\title{
Clinical Features and Treatment Outcomes among Children with Stevens-Johnson Syndrome and Toxic Epidermal Necrolysis: A 20-Year Study in a Tertiary Referral Hospital
}

\author{
Susheera Chatproedprai $(\mathbb{D}$, Vanvara Wutticharoenwong, \\ Therdpong Tempark, and Siriwan Wananukul \\ Department of Pediatrics, Faculty of Medicine, Chulalongkorn University and King Chulalongkorn Memorial Hospital, \\ Bangkok 10330, Thailand
}

Correspondence should be addressed to Susheera Chatproedprai; susheera.c@chula.ac.th

Received 6 January 2018; Revised 20 March 2018; Accepted 2 April 2018; Published 7 May 2018

Academic Editor: Luigi Naldi

Copyright (C) 2018 Susheera Chatproedprai et al. This is an open access article distributed under the Creative Commons Attribution License, which permits unrestricted use, distribution, and reproduction in any medium, provided the original work is properly cited.

\begin{abstract}
Aim. To determine the probable causative factors, clinical features, and treatment outcomes of Stevens-Johnson syndrome (SJS), toxic epidermal necrolysis (TEN), and SJS-TEN overlap in children. Methods. A 20-year database review of all children diagnosed with SJS/TEN/SJS-TEN overlap at the King Chulalongkorn Memorial Hospital, Thailand. Results. 36 patients (M: F, 16:20) with the mean age of $9.2 \pm 4.0$ years were identified. There were 20 cases of SJS, 4 cases of SJS-TEN overlap, and 12 cases of TEN. Drugs were the leading cause for the diseases (72.3\%); antiepileptics were the most common culprits (36.1\%). Cutaneous morphology at presentation was morbilliform rash (83.3\%), blister (38.9\%), targetoid lesions (25.0\%), and purpuric macules (2.8\%). Oral mucosa (97.2\%) and eye (83.3\%) were the 2 most common mucosal involvements. Majority of the cases (77.8\%) were treated with systemic corticosteroids, intravenous immunoglobulin, or both. Treatment outcomes between those who received systemic therapy and those who received only supportive care were comparable. Skin and eye were the principal sites of short-term and long-term complications. Conclusions. SJS/TEN are not common but are serious diseases which lead to significant morbidities in children. Early withdrawal of suspicious causes and meticulous supportive care are very important. This study found that the systemic therapy was not superior to supportive care because the treatment outcomes for both groups were comparable.
\end{abstract}

\section{Introduction}

Stevens-Johnson syndrome (SJS), toxic epidermal necrolysis $(\mathrm{TEN})$, and SJS-TEN overlap are rare but serious diseases. They are considered to be the same spectrum of diseases, defined by the area of epidermal detachment. SJS is the mildest form affecting $<10 \%$ of the body surface area (BSA). TEN is the most severe disease affecting $>30 \%$ of the BSA. Total BSA involvement of $10-30 \%$ is defined as SJS-TEN overlap. The overall incidence of SJS and TEN was 0.4-6 cases per 1,000,000 persons [1, 2].

The optimal treatment for SJS/TEN is inconclusive. Meticulous skin care, hydration, pain control, early identification, and discontinuation of the probable culprit drug as well as an early admission to a specialised unit are the most important things in controlling the disease. Systemic corticosteroids (SCS) and/or intravenous immunoglobulin (IVIG) were proposed systemic treatments but their efficacy remains debatable. The mortality rate is high for SJS and significantly higher for TEN. The reported mortality rate in the Thai population was $7-50 \%$ [3] which is quite higher relative to other countries $[4,5]$.

This study assessed the probable causative factors, clinical features, and treatment outcomes in SJS/TEN/SJS-TEN overlap pediatric patients in a tertiary referral hospital.

\section{Patients and Methods}

This retrospective study was approved by the Institutional Review Board (IRB), Faculty of Medicine, Chulalongkorn University, and adheres to the provisions outlined in the Declaration of Helsinki (IRB number 316/55). 
2.1. Participants. The database for all pediatric inpatients ( $<18$ years old) diagnosed with SJS, TEN, or SJS-TEN, overlap by pediatric dermatologists and admitted to the King Chulalongkorn Memorial Hospital, Bangkok, Thailand, from January 1997 to December 2016 was retrospectively reviewed.

The following data were recorded: (1) history such as demographic data, comorbidity, past medical and allergic history, previous exposure to the inciting drug, and history of concurrent infection, (2) clinical features such as prodromal symptoms, cutaneous lesions (BSA involvement, pattern, and distribution of lesions), number and area of mucosal involvement, (3) treatment such as supportive care (wound care, hydration, and pain control) and/or systemic treatment (SCS and/or IVIG), and (4) outcomes such as duration of hospital stay, short-term complication, and long-term sequel. Long-term sequel was defined as observed end-organ failure after resolution of SJS/TEN, or the onset of another disease during the acute stage which did not resolve at least one month after the resolution of SJS/TEN [6]. Causative drugs were determined by considering the timeline of drug administration and disease occurrence. For the first time exposure, the interval between drug administration and the onset of symptoms should be within a week to a month and less than 2 weeks in the patients with history of re-exposure. In addition, the causative drugs were also identified by the result from the patch test if it was performed.

2.2. Statistical Analysis. Categorical data are presented as number and percentage. Continuous data are expressed as mean and standard deviation (SD). The statistical analysis was performed using Chi-Square test for proportion and Mann-Whitney $U$ test for continuous data. All statistical analyses were done using the SPSS version 20 (IBM Corp, New York, NY, USA). $P$ value $<0.05$ was considered statistically significant.

\section{Results}

A total of 36 patients (male $n=16,44.4 \%$ ) with the mean age (SD) of 9.2 (4.0) years were identified for the study. The age ranged from 1 year 5 months to 15 years 10 months. There were 20 cases of SJS, 4 cases of SJS-TEN overlap, and 12 cases of TEN. The sample size for SJS-TEN overlap patients was small so the authors combined the patients from the SISTEN overlap group with the patients from the TEN group and reclassified them as overlap-TEN group.

3.1. Demographic Data. Fifteen patients (41.6\%) were previously healthy. The remaining 21 cases had underlying diseases. Twelve $(33.3 \%)$ of them had neurological diseases, followed by human immunodeficiency virus $(n=3,8.3 \%)$, end-stage renal disease $(n=2,5.6 \%)$, Wilson disease $(n=$ $2,5.6 \%)$, systemic lupus erythematosus $(n=1,2.8 \%)$, and cyanotic heart disease $(n=1,2.8 \%)$ (Table 1$)$.

3.2. Causative Factors. For majority of the patients, the cause for the disease was prescription drugs $(n=26,72.3 \%)$. The leading culprit was the antiepileptic drugs ( $n=13,36.1 \%)$, followed by antibiotics ( $n=9,25.0 \%)$. In the SJS group, antibiotics (35.0\%) were the leading cause for the disease. In the overlap-TEN group, the most common causes for the disease were antiepileptics (50.0\%) followed by antibiotics (12.5\%).

Almost all patients were investigated to exclude the possible infectious causative factors including Mycoplasma pneumoniae and/or herpes simplex virus and/or Epstein-Barr virus. The results were all negative except for 3 cases. For 1 case (5\%) from the SIS group, infection was the possible cause for the disease but the organism could not be identified. In three cases $(18.8 \%)$ from the overlap-TEN group, infections were the causes of the disease. Two of them had Mycoplasma pneumoniae infection and 1 case had Epstein-Barr virus infection. All of them had no previous history of any drug exposure prior to the lesions. On the other hand, of 36 cases, we could not identify causes of the disease in 6 cases (16.6\%) (Table 1).

Concerning the timeline for considering a drug as suspicious, duration from drug administration to disease occurrence was $12.6 \pm 3.9$ days (7-21 days) for patients with history of first-time exposure and $3.3 \pm 3.8$ days (1-10 days) for those with previous exposure $(P<0.001)$. However, the duration was comparable between SJS group ( $8.6 \pm 6.6$ days) and overlap-TEN group (9.4 \pm 5.0 days).

3.3. Clinical Features. Seventy-five percent of SJS group and $68.8 \%$ of overlap-TEN group had prodromal symptoms. Fever was the main complaint, ranging from 1 to 7 days with the mean duration (SD) of 1.3 (1.5) days, followed by stinging eyes and sore throat. The most common cutaneous morphology at the presentation was morbilliform rash, including maculopapular rash and exanthematous rash (95.0\% in SJS; $68.8 \%$ in overlap-TEN); there were significant differences between the SIS and overlap-TEN groups for morbilliform rash $(P=0.036)$. Purpura was found in only 1 case $(5.0 \%)$ from the SJS group. Five patients (25\%) from the SJS group and 4 cases (25\%) from the overlap-TEN had both blister and morbilliform rash.

The 2 most common areas of mucosal involvement in this study were oral $(n=35,97.2 \%)$ and eye $(n=30$, $83.3 \%$ ) but this was not statistically significant for both groups (Table 2). The number of sites with mucosal involvement varied between 2, 3, and 4 sites. For SJS cases, $45.0 \%(n=9)$ involved 2 mucosal sites, $40.0 \%(n=8)$ involved 3 mucosal sites, and $15.0 \%(n=3)$ involved 4 mucosal sites. For overlapTEN cases, $50.0 \%(n=8)$ involved 2 mucosal sites, $31.2 \%$ $(n=5)$ involved 3 mucosal sites, and $18.8 \%(n=3)$ involved 4 mucosal sites.

Associated abnormalities and visceral organs involvement in this study were predominantly associated with GI abnormalities ( $n=18,50.0 \%)$, especially transaminitis $(n=$ $18,50.0 \%)$ and electrolyte abnormalities $(n=16,44.4 \%)$ but this was not significant between both groups (Table 2).

3.4. Treatment. All cases were treated with multidisciplinary team, meticulous wound care with or without dressings (Acticoat $^{\mathrm{TM}}$, Biobrane $\left.{ }^{\mathrm{TM}}\right)$, hydration, pain control, and isolation at either intensive care unit or a specialised unit as the supportive care. Majority of cases in the SJS group $(n=16$, $80.0 \%)$ and overlap-TEN group $(n=12,75.0 \%)$ were treated 
TABLE 1: Demographics of patients with Stevens-Johnson syndrome and overlap-toxic epidermal necrolysis.

\begin{tabular}{|c|c|c|c|c|}
\hline & SJS, $N=20$ & Overlap-TEN, $N=16$ & Overall, $N=36$ & $P$ value \\
\hline Male (\%) & $8(40.0)$ & $8(50.0)$ & $16(44.4)$ & 0.549 \\
\hline Age $(\mathrm{yr})($ mean $\pm \mathrm{SD})$ & $8.6 \pm 4.2$ & $9.9 \pm 3.6$ & $9.2 \pm 4.0$ & 0.373 \\
\hline Underlying diseases, $N(\%)$ & & & & 0.623 \\
\hline None & $7(35.0)$ & $8(50.0)$ & $15(41.6)$ & \\
\hline $\begin{array}{l}\text { Neurological diseases } \\
\text { (seizure, MELAS, GBS) }\end{array}$ & $6(30.0)$ & $6(37.5)$ & $12(33.3)$ & \\
\hline HIV & $2(10.0)$ & $1(6.2)$ & $3(8.3)$ & \\
\hline ESRD & $1(5.0)$ & $1(6.2)$ & $2(5.6)$ & \\
\hline Wilson disease & $2(10.0)$ & 0 & $2(5.6)$ & \\
\hline SLE & $1(5.0)$ & 0 & $1(2.8)$ & \\
\hline Cyanotic heart disease & $1(5.0)$ & 0 & $1(2.8)$ & \\
\hline Probable causative factors (\%) & & & & 0.185 \\
\hline Unknown & $4(20.0)$ & $2(12.5)$ & $6(16.6)$ & \\
\hline Infection & $1(5.0)$ & $3(18.8)$ & $4(11.1)$ & \\
\hline Drug & $15(75.0)$ & $11(68.7)$ & $26(72.3)$ & \\
\hline Antiepileptics & $5(25.0)$ & $8(50.0)$ & $13(36.1)$ & \\
\hline Antibiotics & $7(35.0)$ & $2(12.5)$ & $9(25.0)$ & \\
\hline D-penicillamine & $2(10.0)$ & 0 & $2(5.6)$ & \\
\hline Antivirus & $1(5.0)$ & $1(6.2)$ & $1(2.8)$ & \\
\hline NSAIDs & 0 & 0 & $1(2.8)$ & \\
\hline
\end{tabular}

MELAS, mitochondrial myopathy, encephalopathy, lactic acidosis, and stroke; GBS, Guillain-Barre syndrome; HIV, human immunodeficiency virus; ESRD, end-stage renal disease; SLE, systemic lupus erythematosus.

TABLE 2: Clinical features of patients with Stevens-Johnson syndrome and overlap-toxic epidermal necrolysis.

\begin{tabular}{|c|c|c|c|c|}
\hline & SJS, $N=20$ & Overlap-TEN, $N=16$ & Overall, $N=36$ & $P$ value* $^{*}$ \\
\hline Prodromal symptoms, N (\%) & $15(75.0)$ & $11(68.8)$ & $26(72.2)$ & 0.677 \\
\hline Fever & $14(70.0)$ & $11(68.8)$ & $25(69.4)$ & 0.352 \\
\hline Stinging eye & $4(20.0)$ & $4(25.0)$ & $8(22.2)$ & 0.477 \\
\hline Sore throat & $5(25.0)$ & $1(6.2)$ & $6(16.7)$ & 0.274 \\
\hline \multicolumn{5}{|l|}{ Cutaneous findings at the presentation, $N(\%)$} \\
\hline Morbilliform rash & $19(95.0)$ & $11(68.8)$ & $30(83.3)$ & 0.036 \\
\hline Blister & $5(25.0)$ & $9(56.3)$ & $14(38.9)$ & 0.056 \\
\hline Targetoid lesions & $6(30.0)$ & $3(18.8)$ & $9(25.0)$ & 0.439 \\
\hline Purpuric macules & $1(5.0)$ & 0 & $1(2.8)$ & 0.364 \\
\hline \multicolumn{5}{|l|}{ Mucosal involvement, $N(\%)$} \\
\hline Oral & $19(95.0)$ & $16(100.0)$ & $35(97.2)$ & 0.364 \\
\hline Eye & $17(85.0)$ & $13(81.2)$ & $30(83.3)$ & 0.764 \\
\hline Genital & $15(75.0)$ & $11(68.8)$ & $26(72.2)$ & 0.677 \\
\hline Anus & $3(15.0)$ & $3(18.8)$ & $6(16.7)$ & 0.764 \\
\hline \multicolumn{5}{|l|}{ Associated abnormalities } \\
\hline GI abnormalities & $9(45.0)$ & $9(56.3)$ & $18(50.0)$ & 0.502 \\
\hline Transaminitis & $9(45.0)$ & $9(56.3)$ & $18(50.0)$ & 0.645 \\
\hline Direct hyperbilirubinemia & $3(15.0)$ & 0 & $3(8.3)$ & 0.106 \\
\hline Electrolyte abnormalities & $8(40.0)$ & $8(50.0)$ & $13(44.4)$ & 0.568 \\
\hline Hyponatremia & $5(25.0)$ & $3(18.8)$ & $8(22.2)$ & 0.654 \\
\hline Hypokalemia & $3(15.0)$ & $4(25.0)$ & $7(19.4)$ & 0.451 \\
\hline Hypocalcemia, hypophosphatemia & 0 & $1(6.2)$ & $1(2.8)$ & 0.257 \\
\hline Renal abnormalities & $1(5.0)$ & $1(6.2)$ & $2(5.6)$ & 0.359 \\
\hline Rising creatinine & $1(5.0)$ & 0 & $1(2.8)$ & 0.364 \\
\hline Rising creatinine and hematuria & 0 & $1(6.2)$ & $1(2.8)$ & 0.257 \\
\hline
\end{tabular}

\footnotetext{
* Significant values are shown in bold.
} 
TABLE 3: Treatment and treatment outcomes of patients with Stevens-Johnson syndrome and overlap-toxic epidermal necrolysis.

\begin{tabular}{|c|c|c|c|c|}
\hline & SJS, $N=20$ & Overlap-TEN, $N=16$ & Overall, $N=36$ & $P$ value* \\
\hline \multicolumn{5}{|l|}{ Treatment, $N(\%)$} \\
\hline Specific treatment & $16(80.0)$ & 75.0 & 77.8 & 0.720 \\
\hline Supportive treatment only & $4(20.00)$ & 25.0 & 22.2 & 0.720 \\
\hline Duration of hospital stay $(\mathrm{d})($ mean $\pm \mathrm{SD})$ & $7.1 \pm 4.1$ & $17.7 \pm 13.1$ & $11.8 \pm 10.6$ & $<0.001$ \\
\hline \multicolumn{5}{|l|}{ Comorbidities/short-term complication, $N(\%)$} \\
\hline Skin & $10(50.0)$ & $16(100.00)$ & $26(72.2)$ & 0.001 \\
\hline Dyspigmentation & $10(50.0)$ & $16(100.00)$ & $26(72.2)$ & 0.001 \\
\hline Nail change & 0 & $4(25.00)$ & $4(11.1)$ & 0.018 \\
\hline Eye & $10(50.0)$ & $14(87.5)$ & $24(66.7)$ & 0.018 \\
\hline Conjunctivitis & $8(40.0)$ & $9(56.3)$ & $17(47.2)$ & 0.332 \\
\hline Corneal epithelial defects & $2(10.0)$ & $4(25.0)$ & $6(16.7)$ & 0.230 \\
\hline Synechiae/symblepharon & $2(10.0)$ & $4(25.0)$ & $6(16.7)$ & 0.248 \\
\hline Pseudomembrane & $3(15.0)$ & $3(18.8)$ & $6(16.7)$ & 0.764 \\
\hline Superinfection & $3(15.0)$ & $7(43.8)$ & $10(27.8)$ & 0.053 \\
\hline Vaginal adhesion & 0 & $3(18.8)$ & $3(8.3)$ & 0.043 \\
\hline Pneumonia & $1(5.0)$ & $1(6.2)$ & $2(5.6)$ & 0.871 \\
\hline Pancreatitis & 0 & $1(6.2)$ & $1(2.8)$ & 0.257 \\
\hline Adrenal insufficiency & 0 & $1(6.2)$ & $1(2.8)$ & 0.257 \\
\hline \multicolumn{5}{|l|}{ Long-term sequel, $N(\%)$} \\
\hline Skin & $5(25.0)$ & $14(87.5)$ & $19(52.8)$ & 0.001 \\
\hline Eye & $4(20.0)$ & $9(56.3)$ & $13(36.1)$ & 0.042 \\
\hline GI (transaminitis) & $2(10.0)$ & $6(37.5)$ & $8(22.2)$ & 0.124 \\
\hline Recurrence, N (\%) & $1(5.0)$ & 0 & $1(2.8)$ & 0.364 \\
\hline Mortality rate, $N(\%)$ & 0 & 0 & 0 & \\
\hline
\end{tabular}

* Significant values are shown in bold.

with systemic treatment (systemic corticosteroids (SCS) at the dose equivalent to prednisolone $1-4 \mathrm{mg} / \mathrm{kg} / \mathrm{day}$, intravenous immunoglobulin (IVIG) at the total dose $2-7 \mathrm{mg} / \mathrm{kg}$, or both). All 16 cases from the SJS group, who received systemic treatment, were treated with SCS. The duration of SCS treatment including tapering period varied from 7 to 60 days with the mean (SD) of 22.9 (14.5) days.

In regard to the overlap-TEN group who received systemic treatment $(n=12), 8$ cases $(66.7 \%)$ were treated with SCS alone, 1 case $(8.3 \%)$ was treated with IVIG only at the dose of $1 \mathrm{mg} / \mathrm{kg} /$ day for 4 days, and 3 cases (25.0\%) were treated with both SCS and IVIG. Of these 3 cases treated with both SCS and IVIG, 1 case with severe epidermal detachment (>90\% BSA) received IVIG $1 \mathrm{mg} / \mathrm{kg} /$ day for 3 days and SCS at the dose equivalent to prednisolone $4 \mathrm{mg} / \mathrm{kg} / \mathrm{day}$ but the degree of skin detachment still progressed so additional IVIG $2 \mathrm{mg} / \mathrm{kg} /$ day for 2 days was prescribed. The duration of SCS treatment in this group ranged from 4 to 69 days with the mean (SD) of 39.3 (21.0) days. There was no significant difference between the duration of SCS treatment between the SJS group and the overlap-TEN group $(P=0.171)$.

3.5. Treatment Outcomes. The duration of hospital stay was significantly different between the SJS $(7.1 \pm 4.1$ days $)$ and overlap-TEN groups $(17.7 \pm 13.1$ days $)(P<0.001)$. Also, the overall short-term eye complication was significantly different between the SJS ( $n=10,50.0 \%)$ and overlap-TEN groups $(n=14,87.5 \%)(P=0.018)$. In addition, skin change (dyspigmentation), nail change, and vaginal adhesion were significantly different between the SIS and overlap-TEN groups. Other outcomes such as prevalence of superinfection, pneumonia, pancreatitis, and adrenal insufficiency were comparable between the SJS and overlap-TEN groups (Table 3). No case of the SIS group had pancreatitis or adrenal insufficiency where the overlap-TEN group had 1 case $(6.2 \%$, $P=0.257)$ of each.

The mean duration of follow-up was 13.1 months (SD 19.9, 1-80 months). In regard to long-term skin sequel, dyspigmentation was the most common finding in the SJS $(n=4,20.0 \%)$ and overlap-TEN $(n=14,87.5 \%)$ groups $(P<0.001)$. One case $(5.0 \%)$ from the SJS group had xerosis and 1 case $(6.2 \%)$ from the overlap-TEN group had nail loss. For long-term eye sequel, dry eye was the single long-term complication found in the SJS group $(n=4,20.0 \%)$, while, in the overlap-TEN group, dry eye was the most common longterm sequel $(n=6,37.5 \%)$, followed by corneal scar $(n=4$, $25.0 \%)$, keratopathy $(n=3,18.8 \%)$, and subconjunctival fibrosis ( $n=1,6.2 \%)$. Transaminitis was the long-term GI problem found in the SJS group $(n=2,10.0 \%)$ and overlapTEN group $(n=6,37.4 \%)$ (Table 4$)$. There was no deceased SJS/TEN case in this study. Recurrence occurred in only 1 case from the SJS group (5.0\%). 
TABLE 4: Long-term sequel according to diagnosis and treatment.

\begin{tabular}{|c|c|c|c|c|c|c|}
\hline & \multicolumn{3}{|c|}{ Diagnosis } & \multicolumn{3}{|c|}{ Treatment } \\
\hline & SJS, $N=20$ & Overlap-TEN, $N=16$ & $P$ value ${ }^{*}$ & Supportive care, $N=8$ & Systemic treatment, $N=28$ & $P$ value* $^{*}$ \\
\hline \multicolumn{7}{|l|}{ Long-term skin sequel, $N(\%)$} \\
\hline Dyspigmentation & $4(20.0)$ & $14(87.5)$ & $<0.001$ & $3(37.5)$ & $15(53.6)$ & 0.423 \\
\hline Xerosis & $1(5.0)$ & 0 & 0.364 & $1(12.5)$ & 0 & 0.058 \\
\hline Nail loss & 0 & $1(6.2)$ & 0.257 & 0 & $1(3.6)$ & 0.588 \\
\hline \multicolumn{7}{|l|}{ Long-term eye sequel, $N(\%)$} \\
\hline Dry eye & $4(20.0)$ & $6(37.5)$ & 0.244 & $4(50.0)$ & $6(21.6)$ & 0.112 \\
\hline Corneal scar & 0 & $4(25.0)$ & 0.018 & $1(12.5)$ & $3(10.7)$ & 0.887 \\
\hline Keratopathy & 0 & $3(18.8)$ & 0.043 & $2(25.0)$ & $1(3.6)$ & 0.053 \\
\hline Subconjunctival fibrosis & 0 & $1(6.2)$ & 0.257 & 0 & $1(3.6)$ & 0.588 \\
\hline \multicolumn{7}{|l|}{ Long-term GI sequel, N (\%) } \\
\hline \multicolumn{7}{|l|}{ Transaminitis } \\
\hline Less than $3 \mathrm{~m}$ & $2(10.0)$ & $5(31.2)$ & 0.109 & $2(25.0)$ & $5(17.9)$ & 0.653 \\
\hline More than $3 \mathrm{~m}$ & 0 & $1(6.2)$ & 0.257 & 0 & $1(3.6)$ & 0.588 \\
\hline
\end{tabular}

${ }^{*}$ Significant values are shown in bold.

TABLE 5: Treatment outcomes according to supportive care alone versus systemic treatment.

\begin{tabular}{|c|c|c|c|}
\hline & Supportive care only, $N=8$ & Systemic treatment, $N=28$ & $P$ value \\
\hline Length of hospital stay $(\mathrm{d})$ (mean $\pm \mathrm{SD})$ & $9.9 \pm 7.4$ & $12.3 \pm 11.4$ & 0.593 \\
\hline \multicolumn{4}{|c|}{ Comorbidities/short-term complication, $N(\%)$} \\
\hline \multicolumn{4}{|l|}{ Skin } \\
\hline Dyspigmentation & $5(62.5)$ & $21(75.0)$ & 0.486 \\
\hline Nail change & 0 & $4(14.3)$ & 0.257 \\
\hline Eye & $5(62.5)$ & $19(67.9)$ & 0.777 \\
\hline Conjunctivitis & $3(37.5)$ & $14(50.0)$ & 0.532 \\
\hline Corneal epithelial defects & $2(25.0)$ & $4(14.3)$ & 0.473 \\
\hline Synechiae/symblepharon & $2(25.0)$ & $4(14.3)$ & 0.608 \\
\hline Pseudomembrane & $1(12.5)$ & $5(17.9)$ & 0.720 \\
\hline Superinfection & $1(12.5)$ & $9(32.1)$ & 0.532 \\
\hline Vaginal adhesion & $1(12.5)$ & $2(7.1)$ & 0.629 \\
\hline Pneumonia & $1(12.5)$ & $1(3.6)$ & 0.331 \\
\hline Pancreatitis & 0 & $1(3.6)$ & 0.588 \\
\hline Adrenal insufficiency & 0 & $1(3.6)$ & 0.588 \\
\hline \multicolumn{4}{|l|}{ Long-term sequel, $N(\%)$} \\
\hline Skin & $4(50.0)$ & $15(53.6)$ & 0.261 \\
\hline Eye & $5(62.5)$ & $8(28.6)$ & 0.302 \\
\hline GI (transaminitis) & $2(25.0)$ & $6(21.4)$ & 0.795 \\
\hline Recurrence, N (\%) & 0 & $1(3.6)$ & 0.588 \\
\hline
\end{tabular}

Comparing between those receiving only supportive care $(n=8,22.2 \%)$ and those receiving systemic treatment $(n=$ $28,77.8 \%)$, mean (SD) of duration of hospital stay was 9.9 (7.4) and $12.3(11.4)(P=0.593)$. Overall short-term and long-term complications were comparable between both groups without significant differences (Table 5).

Comparisons of clinical features, laboratory findings, and treatment outcomes between children in this study and adults from previous studies were shown in Table 6.

\section{Discussion}

This study confirms the rarity of SJS/TEN cases. For our institution, a main tertiary referral hospital in Thailand, the prevalence was 1.8 cases/year. This finding is similar to other previous studies; there was a slightly higher risk for girls and a higher number of chronic health conditions associated with SJS cases $[20,21]$ in contrast to overlap-TEN patients. Drugs were the leading causative factors for both 
TABLE 6: Comparison of clinical features and treatment outcomes between children and adults with Stevens-Johnson syndrome and toxic epidermal necrolysis.

\begin{tabular}{|c|c|c|}
\hline & Children & Adult \\
\hline \multicolumn{3}{|l|}{ Sex $\%$} \\
\hline Male & 44.4 & $42.1-58.3[6-13]$ \\
\hline Mean age (yr) & 9.2 & $40.1-56.6[6-13]$ \\
\hline Underlying disease, $\%$ & 58.4 & $33.3-76.9[8,11-14]$ \\
\hline \multicolumn{3}{|l|}{ Causative factor, $\%$} \\
\hline Drug-related & 72.3 & $52.4-100.0[7-9,11-13]$ \\
\hline Non-drug-related & 27.7 & $0-47.6[7-9,11-13]$ \\
\hline \multicolumn{3}{|l|}{ Prodromal symptoms, \% } \\
\hline Fever & 69.4 & $59.8-94.7[11,13,14]$ \\
\hline \multicolumn{3}{|l|}{ Mucosal involvement, \% } \\
\hline Oral & 97.2 & $38.6-85.4[7,8,11,13]$ \\
\hline Eye & 83.3 & $59.8-64.4[7,8,11,13]$ \\
\hline Genital & 72.2 & $32.9-41.4[7,8,11,13]$ \\
\hline Anus & 16.7 & $\mathrm{n} / \mathrm{a}$ \\
\hline Nose & - & $3.6[11]$ \\
\hline \multicolumn{3}{|l|}{ Associated abnormalities, \% } \\
\hline GI (Liver) abnormalities & 50.0 & $36.4-48.8[7,8,11]$ \\
\hline Renal abnormalities & 5.6 & 9.1-17.1 [7, 8, 11] \\
\hline Lung abnormalities & - & $11.4[8]$ \\
\hline Encephalopathy & - & $8.0[7,8]$ \\
\hline \multicolumn{3}{|l|}{ Treatment, $\%$} \\
\hline Specific treatment & 77.8 & $46.6-97.7[7-9,11-13]$ \\
\hline Supportive care only & 22.2 & $2.3-53.4[7-9,11-13]$ \\
\hline Duration of hospital stay (d) & 11.8 & $10.0-37.0[6-9,11-14]$ \\
\hline \multicolumn{3}{|l|}{ Short-term complication, \% } \\
\hline \multicolumn{3}{|l|}{ Skin } \\
\hline Dyspigmentation & 72.2 & $13.7-69.0[6,12]$ \\
\hline Nail change/loss & 11.1 & $2.9-46.0[6,7,12]$ \\
\hline Eye & 66.7 & $0-69.2[7,11,12]$ \\
\hline Conjunctivitis & 47.2 & $\mathrm{n} / \mathrm{a}$ \\
\hline Corneal epithelial defects & 16.7 & Unidentified [11] \\
\hline Synechiae/symblepharon & 16.7 & Unidentified [11] \\
\hline Pseudomembrane & 16.7 & $4.8[11]$ \\
\hline Superinfection & 27.8 & $8.0-56.2[7,8,14]$ \\
\hline Vaginal adhesion & 8.3 & $7.7[12]$ \\
\hline Pneumonia & 5.6 & $9.2[7]$ \\
\hline Pancreatitis & 2.8 & Cases report [15-17] \\
\hline Adrenal insufficiency & 2.8 & Case report [18] \\
\hline \multicolumn{3}{|l|}{ Long-term sequel, \% } \\
\hline \multicolumn{3}{|l|}{ Skin } \\
\hline Dyspigmentation & 50.0 & $13.7-69.0[6,12]$ \\
\hline Xerosis & 2.8 & $\mathrm{n} / \mathrm{a}$ \\
\hline Nail loss & 2.8 & $1.1[7]$ \\
\hline Eye & 36.1 & $9.8-77.0[6,11,12,19]$ \\
\hline Dry eye & 27.8 & $31.0-32.4[6,12]$ \\
\hline Corneal scar & 11.1 & $4.9-15.4[6,12]$ \\
\hline Keratopathy & 8.3 & $\mathrm{n} / \mathrm{a}$ \\
\hline Subconjunctival fibrosis & 2.8 & $\mathrm{n} / \mathrm{a}$ \\
\hline Mortality rate, $\%$ & 0 & $6.8-34.4[6-9,11-14]$ \\
\hline
\end{tabular}

n/a, not available. 
groups. These results are consistent with prior findings [2224]. Antiepileptics were the most common culprit drugs for the overlap-TEN group in this study. It is thought that a reactive drug metabolite exerts a direct effect on the keratinocytes [25]. CD8+ T cells, stimulated by the causative drugs or drug metabolites, mediate keratinocyte apoptosis by at least 3 different pathways: (1) Fas/Fas ligand interaction, (2) cytotoxic T-cell, and (3) natural killer- (NK-) cell damage via perforin/granzyme $\mathrm{B} /$ granulysin, and tumor necrosis factor$\alpha$ (TNF- $\alpha)[26,27]$. In this study, 2 cases with TEN had mycoplasma infection without the previous history of any drug exposure whereas, in other studies, only SJS cases had mycoplasma infection [23]. We did not assume that these 2 cases had mycoplasma induced rash and mucositis [28] because both of them had severe clinical courses which needed 22 and 55 days of admission and both of them encountered long-term eye sequel. There was no report of herpes simplex virus (HSV) infection in this study which contradicts previous reports that there was 9.0-19.7\% of HSV infection among SJS patients [24, 29].

The most common cutaneous findings at the presentation in this study were morbilliform rash, defined as maculopapular rash or exanthematous rash, but excluding atypical targetoid macules or purpuric macules. One-fourth of cases from the SJS group and $56.3 \%$ cases $(n=9)$ from the overlapTEN group had blisters. The difference in the prevalence of blisters in overlap-TEN group was 2.25 times more common than in SJS group but it did not reach statistical significance. The combination of SJS-TEN overlap cases with TEN cases in this study may explain the skewness of the trend. Mucosal involvement was predominant at the oral mucosa (95.0-100.0\%), followed by eye (81.2-85.0\%), genitalia (68.6-75.0\%), and anus (15.0-18.8\%). Lesions at more than 2 sites were $50.0-55.0 \%$. The prevalence of ocular involvement, genital involvement, and lesions at more than 2 sites was slightly higher than the previous data [7, 23].

The prevalence of mucosal involvement was comparable between the SJS and over-TEN groups. However, its severity of involvement leading to either short-term complication or long-term sequel was statistically significant in overlapTEN groups, especially for the eye $(P=0.018)$ and genital involvement $(P=0.043)$.

No case with encephalopathy was observed in the present study in contrast to Yamane's study which found 3.8\% in SIS and $14.3 \%$ in TEN cases [7].

For treatments, systemic corticosteroids were mainly used in both groups. SCS treatment can decrease the percentage of perforin-positive CD8+ T lymphocytes [30] and decrease excessive immune response [7]. SCS has been suggested to be a valid treatment $[31,32]$ for the disease but the result of this study did not support the efficacy of SCS treatment over the supportive care alone. The duration of hospital stay, short-term and long-term sequel, and recurrence were comparable between systemic treatment group and the supportive care alone group. Although the prevalence of longterm eye sequel in cases with supportive care alone $(62.5 \%)$ was 2.18 times higher than in the systemic treatment $(28.6 \%)$ group, this was not statistically significant $(P=0.302)$. There was no deceased case in this study.
As for the IVIG therapy, only 1 patient in this study was treated with IVIG alone. Additional 3 cases were treated with both IVIG and SCS. Because few were treated with IVIG, therefore we cannot assess the treatment outcome of IVIG to be positive or negative. However, there were studies documenting the favourable outcome of IVIG either alone or combined with SCS in slowing the disease progression among SJS/TEN patients $[23,33,34]$.

Ophthalmic complications were found in SJS/TEN with the incidence ranging from $20.0-81.0 \%$ [4, 6, 35-37]. In this study, short-term complications were seen in $66.7 \%$ of cases and long-term complications were seen in $36.1 \%$ of cases. The incidence of these complications was significantly higher in overlap-TEN group than in the SJS group for both shortterm $(P=0.018)$ and long-term $(P=0.042)$ complications. In addition, this trend was significantly observed in skin dyspigmentation $(P=0.001)$, nail change $(P=0.018)$, and vaginal adhesion $(P=0.043)$.

Other complications were rare but were present in this study. A case with TEN had adrenal insufficiency and another case with SJS-TEN overlap had pancreatitis. Both of them were previously healthy and had no prior medical exposure to anything that can cause the complication. Mycoplasma pneumoniae was presumed to be the cause for the case with adrenal insufficiency by positive IgM serology to Mycoplasma pneumoniae. Epstein-Barr virus was the cause for the case with pancreatitis by positive IgM serology to viral capsid antigen. It has been reported that adrenal insufficiency and tuberculosis in an adult were associated with SJS [18]. To our knowledge, there has been no report of TEN associated with adrenal insufficiency in children. In regard to the pancreatitis, there have been only 2 reports associated with TEN and SJS in children $[38,39]$ and few reports in adults [15-17].

Comparing to adults' data from previous studies [6$14,19]$, children tend to have higher frequency of mucosal involvement in all areas including short-term dyspigmentation. However, renal abnormalities were less frequent and there were no lung abnormalities, encephalopathy, or mortality in this study.

The limitation in the present study was that the data was from a single referral centre. Another limitation was its retrospective design. Therefore, we cannot identify the causative factors for all patients. In addition, there were few cases that were treated with IVIG so that we cannot make any assumption toward its treatment outcome. However, it is difficult and unethical to perform a randomized controlled trial for these diseases.

\section{Conclusion}

SJS/TEN are serious diseases even though they are rare diseases. Compared to the SJS group, the overlap-TEN group had more significant morbidities of the skin, eye, and genital organs. Early and prompt recognition, early withdrawal of suspicious causative factors, meticulous supportive care, and an early admission to a specialised unit are the most essential parts of managing these patients. In this study, the treatment outcomes were comparable between systemic treatment and 
supportive care only. This indicated that systemic treatment was not superior to supportive care only.

\section{Disclosure}

Part of this study was presented as a poster presentation at the 23rd World Congress of Dermatology, Vancouver, Canada.

\section{Conflicts of Interest}

The authors have no conflicts of interest to declare.

\section{Acknowledgments}

The authors would like to gratefully acknowledge the Chulalongkorn University research affairs team for English editing of this manuscript.

\section{References}

[1] B. Rzany, M. Mockenhaupt, S. Baur et al., "Epidemiology of erythema exsudativum multiforme majus, Stevens-Johnson syndrome, and toxic epidermal necrolysis in Germany (1990-1992): Structure and results of a population-based registry," Journal of Clinical Epidemiology, vol. 49, no. 7, pp. 769-773, 1996.

[2] J. C. Roujeau and R. S. Stern, "Severe adverse cutaneous reactions to drugs," The New England Journal of Medicine, vol. 331, no. 19, pp. 1272-1285, 1994.

[3] P. Dilokthornsakul, R. Sawangjit, C. Inprasong et al., "Healthcare utilization and cost of Stevens-Johnson syndrome and toxic epidermal necrolysis management in Thailand," Journal of Postgraduate Medicine, vol. 62, no. 2, pp. 109-114, 2016.

[4] R. Gerull, M. Nelle, and T. Schaible, "Toxic epidermal necrolysis and Stevens-Johnson syndrome: A review," Critical Care Medicine, vol. 39, no. 6, pp. 1521-1532, 2011.

[5] T. Harr and L. E. French, "Toxic epidermal necrolysis and Stevens-Johnson syndrome," Orphanet Journal of Rare Diseases, vol. 5, no. 1, article no. 39, 2010.

[6] C.-W. Yang, Y.-T. Cho, K.-L. Chen, Y.-C. Chen, H.-L. Song, and C.-Y. Chu, "Long-term sequelae of stevens-johnson syndrome/toxic epidermal necrolysis," Acta Dermato-Venereologica, vol. 96, no. 4, pp. 525-529, 2016.

[7] Y. Yamane, S. Matsukura, Y. Watanabe et al., "Retrospective analysis of Stevens-Johnson syndrome and toxic epidermal necrolysis in 87 Japanese patients - Treatment and outcome," Allergology International, vol. 65, no. 1, pp. 74-81, 2016.

[8] L. Wang and X.-L. Mei, "Retrospective analysis of stevens-johnson syndrome and toxic epidermal necrolysis in 88 Chinese patients," Chinese Medical Journal, vol. 130, no. 9, pp. 1062-1068, 2017.

[9] J. Thammakumpee and S. Yongsiri, "Characteristics of toxic epidermal necrolysis and stevens-johnson syndrome: A 5year retrospective study," Journal of the Medical Association of Thailand, vol. 96, no. 4, pp. 399-406, 2013.

[10] M. A. Miliszewski, M. G. Kirchhof, S. Sikora, A. Papp, and J. P. Dutz, "Stevens-Johnson Syndrome and Toxic Epidermal Necrolysis: An Analysis of Triggers and Implications for Improving Prevention," American Journal of Medicine, vol. 129, no. 11, pp. 1221-1225, 2016.

[11] H.-I. Kim, S.-W. Kim, G.-Y. Park et al., "Causes and treatment outcomes of Stevens-Johnson syndrome and toxic epidermal necrolysis in 82 adult patients," Korean Journal of Internal Medicine, vol. 27, no. 2, pp. 203-210, 2012.

[12] J. Haber, W. Hopman, M. Gomez, and R. Cartotto, "Late outcomes in adult survivors of toxic epidermal necrolysis after treatment in a burn center," Journal of Burn Care \& Rehabilitation, vol. 26, no. 1, pp. 33-41, 2005.

[13] B. Gerdts, A. F. P. M. Vloemans, and R. W. Kreis, "Toxic epidermal necrolysis; 15.yearsexperience in a Dutch burns centre," Journal of the European Academy of Dermatology and Venereology, vol. 21, no. 6, pp. 781-788, 2007.

[14] G. Gravante, D. Delogu, M. Marianetti, M. Trombetta, G. Esposito, and A. Montone, "Toxic epidermal necrolysis and Steven Johnson syndrome: 11-years experience and outcome," European Review for Medical and Pharmacological Sciences, vol. 11, no. 2, pp. 119-127, 2007.

[15] H. Tagami and K. Iwatsuki, "Elevated serum amylase in toxic epidermal necrolysis," British Journal of Dermatology, vol. 115, no. 2, pp. 250-250, 1986.

[16] M. Coetzer, A. E. Van Der Merwe, and B. L. Warren, "Toxic epidermal necrolysis in a burn patient complicated by acute pancreatitis," Burns, vol. 24, no. 2, pp. 181-183, 1998.

[17] F. M. Tatnall, H. J. Dodd, and I. Sarkany, "Elevated serum amylase in a case of toxic epidermal necrolysis," British Journal of Dermatology, vol. 113, no. 5, pp. 629-630, 1985.

[18] W. Wermut and A. Kubasik, "Erythema muldforme, addison's disease; and stevens-johnson syndrome," British Medical Journal, vol. 3, no. 5825, p. 531, 1972.

[19] J. Wilkins, L. Morrison, and C. R. White Jr., "Oculocutaneous manifestations of the erythema multiforme/Stevens-Johnson syndrome/toxic epidermal necrolysis spectrum," Dermatologic Clinics, vol. 10, no. 3, pp. 571-582, 1992.

[20] D. Y. Hsu, J. Brieva, N. B. Silverberg, A. S. Paller, and J. I. Silverberg, "Pediatric Stevens-Johnson syndrome and toxic epidermal necrolysis in the United States," Journal of the American Academy of Dermatology, vol. 76, no. 5, pp. 811-817.e4, 2017.

[21] J. Diphoorn, S. Cazzaniga, C. Gamba et al., "Incidence, causative factors and mortality rates of Stevens-Johnson syndrome (SJS) and toxic epidermal necrolysis (TEN) in northern Italy: Data from the REACT registry," Pharmacoepidemiology and Drug Safety, vol. 25, no. 2, pp. 196-203, 2016.

[22] F. Zhang and J. Zhou, “Toxic epidermal necrolysis: 13 years of experience in the management at a Department of Dermatology in China," Cutaneous and Ocular Toxicology, vol. 36, no. 1, pp. 19-24, 2017.

[23] M. J.-A. Koh and Y.-K. Tay, "Stevens-Johnson syndrome and toxic epidermal necrolysis in Asian children," Journal of the American Academy of Dermatology, vol. 62, no. 1, pp. 54-60, 2010.

[24] Y. Finkelstein, G. S. Soon, P. Acuna et al., "Recurrence and outcomes of Stevens-Johnson syndrome and toxic epidermal necrolysis in children," Pediatrics, vol. 128, no. 4, pp. 723-728, 2011.

[25] A. Nassif, A. Bensussan, L. Boumsell et al., "Toxic epidermal necrolysis: Effector cells are drug-specific cytotoxic T cells," The Journal of Allergy and Clinical Immunology, vol. 114, no. 5, pp. 1209-1215, 2004.

[26] M. J.-A. Koh and Y.-K. Tay, "An update on Stevens-Johnson syndrome and toxic epidermal necrolysis in children," Current Opinion in Pediatrics, vol. 21, no. 4, pp. 505-510, 2009.

[27] R. A. Schwartz, P. H. McDonough, and B. W. Lee, "Toxic epidermal necrolysis: part I. Introduction, history, classification, clinical features, systemic manifestations, etiology, and 
immunopathogenesis," Journal of the American Academy of Dermatology, vol. 69, no. 2, pp. 173.e1-173.e13, 2013.

[28] T. N. Canavan, E. F. Mathes, I. Frieden, and K. Shinkai, "Mycoplasma pneumoniae-induced rash and mucositis as a syndrome distinct from Stevens-Johnson syndrome and erythema multiforme: A systematic review," Journal of the American Academy of Dermatology, vol. 72, no. 2, pp. 239-245.e4, 2015.

[29] R. Forman, G. Koren, and N. H. Shear, "Erythema multiforme, Stevens-Johnson syndrome and toxic epidermal necrolysis in children: A review of 10 years' experience," Drug Safety, vol. 25, no. 13, pp. 965-972, 2002.

[30] S. J. Posadas, A. Padial, M. J. Torres et al., "Delayed reactions to drugs show levels of perforin, granzyme B, and Fas-L to be related to disease severity," The Journal of Allergy and Clinical Immunology, vol. 109, no. 1, pp. 155-161, 2002.

[31] A. Tripathi, A. M. Ditto, L. C. Grammer et al., "Corticosteroid therapy in an additional 13 cases of Stevens-Johnson syndrome: a total series of 67 cases." Allergy and asthma proceedings : the official journal of regional and state allergy societies, vol. 21, no. 2, pp. 101-105, 2000.

[32] K. Hirahara, Y. Kano, Y. Sato et al., "Methylprednisolone pulse therapy for Stevens-Johnson syndrome/toxic epidermal necrolysis: Clinical evaluation and analysis of biomarkers," Journal of the American Academy of Dermatology, vol. 69, no. 3, pp. 496498, 2013.

[33] S. Jagadeesan, K. Sobhanakumari, S. Sadanandan et al., "Low dose intravenous immunoglobulins and steroids in toxic epidermal necrolysis: A prospective comparative open-labelled study of 36 cases," Indian Journal of Dermatology, Venereology and Leprology, vol. 79, no. 4, pp. 506-511, 2013.

[34] L.-P. Ye, C. Zhang, and Q.-X. Zhu, "The effect of intravenous immunoglobulin combined with corticosteroid on the progression of stevens-Johnson syndrome and toxic epidermal necrolysis: A meta-Analysis," PLoS ONE, vol. 11, no. 11, Article ID e0167120, 2016.

[35] C. J. Catt, G. M. Hamilton, J. Fish, K. Mireskandari, and A. Ali, "Ocular Manifestations of Stevens-Johnson Syndrome and Toxic Epidermal Necrolysis in Children," American Journal of Ophthalmology, vol. 166, pp. 68-75, 2016.

[36] R. A. Schwartz, P. H. McDonough, and B. W. Lee, "Toxic epidermal necrolysis: Part II. Prognosis, sequelae, diagnosis, differential diagnosis, prevention, and treatment," Journal of the American Academy of Dermatology, vol. 69, no. 2, pp. 187-e16, 2013.

[37] S. Alerhand, C. Cassella, and A. Koyfman, "Stevens-Johnson Syndrome and Toxic Epidermal Necrolysis in the Pediatric Population: A Review," Pediatric Emergency Care, vol. 32, no. 7, pp. 472-478, 2016.

[38] M. L. Dylewski, K. Prelack, T. Keaney, and R. L. Sheridan, "Asymptomatic hyperamylasemia and hyperlipasemia in pediatric patients eith toxic epidermal necrolysis," Journal of Burn Care \& Research, vol. 31, no. 2, pp. 292-296, 2010.

[39] M. Garcia, M. J. Mhanna, M.-J. Chung-Park, P. H. Davis, and M. D. Srivastava, "Efficacy of early immunosuppressive therapy in a child with carbamazepine-associated vanishing bile duct and Stevens-Johnson syndromes," Digestive Diseases and Sciences, vol. 47, no. 1, pp. 177-182, 2002. 


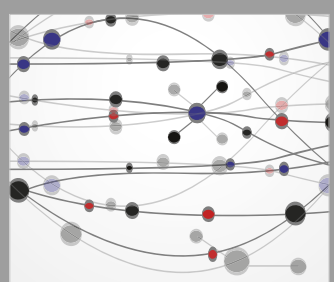

The Scientific World Journal
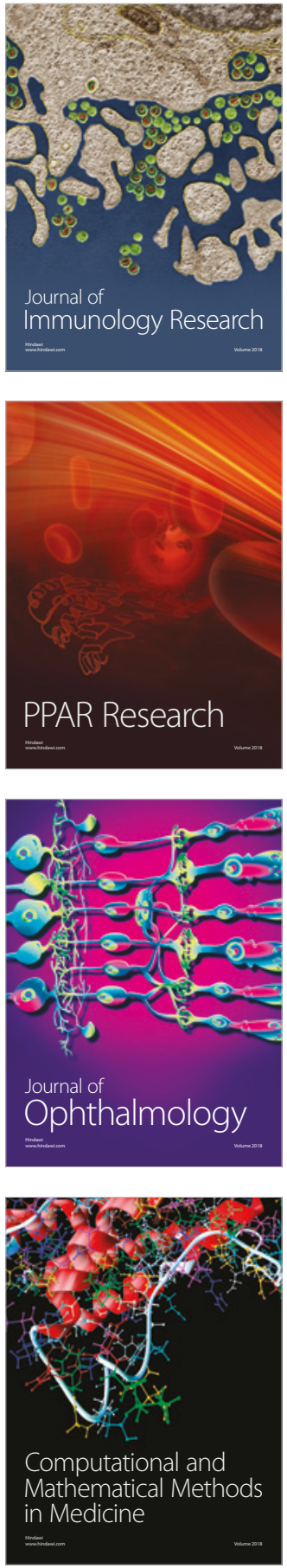

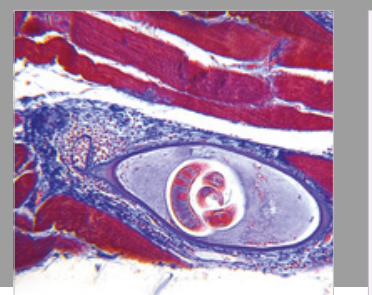

Gastroenterology Research and Practice

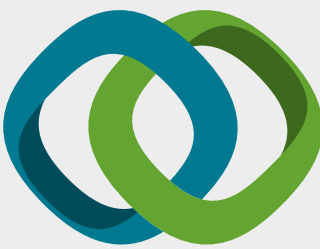

\section{Hindawi}

Submit your manuscripts at

www.hindawi.com
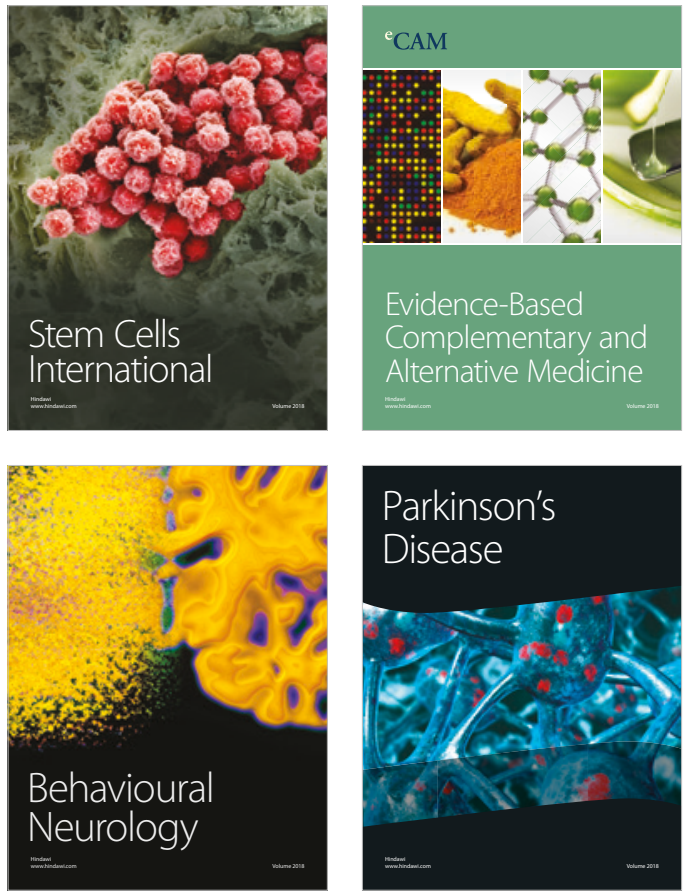

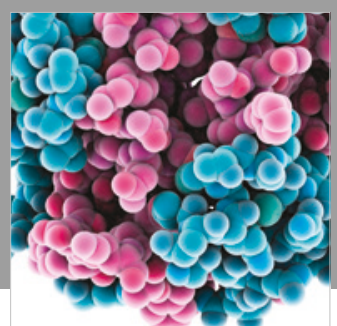

ournal of

Diabetes Research

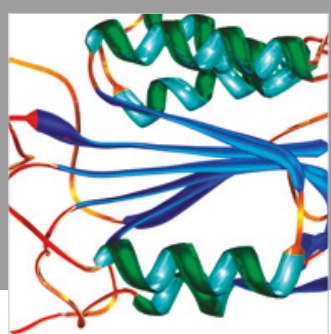

Disease Markers
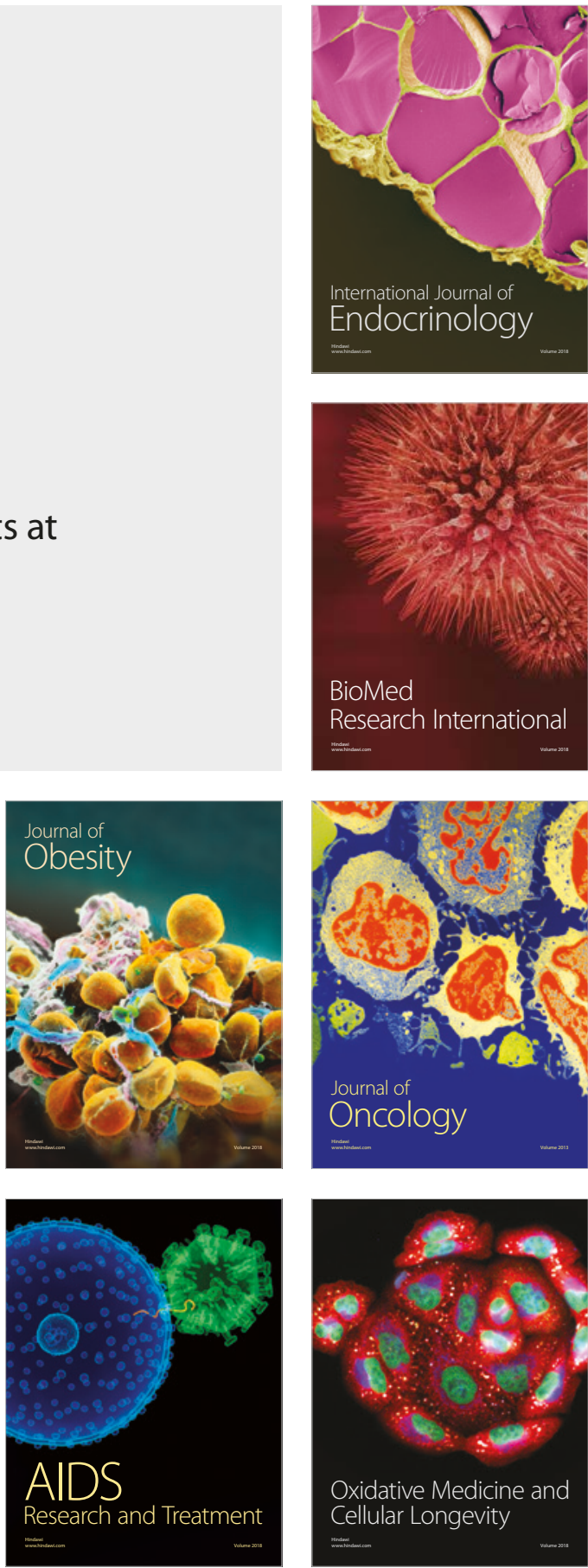